\title{
Editorial
}

\section{Artificial Intelligence in Dentistry}

Artificial Intelligence (AI) sometimes called machine intelligence is the intelligence expressed by machines in contrast to the natural intelligence expressed by humans and animals. From past decades technologies have been developing to mimic the functions of a human brain. In 1950, a breakthrough occurred when a British mathematician, Alan Turing devised a machine to decode encrypted messages. ${ }^{1}$ In 1956 conference, researchers from Dartmouth College introduced programs that were described as 'astonishing' in which computers could learn—checker strategies, solving word problems in algebra, proving logical theorems and speaking English. ${ }^{2}$ Later in 1990s, AI began to be used for logistics, data mining, medical diagnosis and other areas.

Advantages of AI are: It made the dental treatments affordable, efficient and personalized. Dentists will be able to pinpoint specific problems thereby speeding the recovery. ${ }^{3}$ It may not save time but can provide patients accurate diagnosis. Watson was declared as a hero after successful diagnosis of leukemia. ${ }^{4}$ It helped the healthcare professionals to analyse and deliver informations more effectively than the human assistants and could overcome the communication gap. ${ }^{5}$ Along with images obtained from X-ray machines, they will be able to get preliminary analysis of those images.

AI has got certain disadvantages as well. Legal questions may arise on giving power to a machine to control or influence the health of a human. The system can never replace human expertise as humans are required to frequently monitor and update systems. ${ }^{6}$ Machines will require regular maintainance and the cost needed to fix the setup would be high. Machines may have ability to store a large amount of data but can never be compared to human brain. Also, Machines would not be able to alter their responses in changing situations as they function in the manner they are programed. ${ }^{5}$ Human beings are emotional intellectuals and can think, feel and use their senses to come to a solution. But the major disadvantage of a machine is that they lack the empathy and care. ${ }^{3}$ Applications of artificial intelligence as follows: they had made the innovation of self driving trucks possible although they are in the testing phase; banks use AI to organize operations; maintain book keeping; invest in stocks and manage properties. They can also react to changes overnight or when the business is not taking place. In the field of medicine, they are used in disease identification and diagnosis, personalized treatment and behavioral modification, drug discovery and manufacturing, can keep the health records and also used in epidemic outbreak prediction. Dentistry also make use of $\mathrm{AI}$ in a wide range of actions. They include booking appointments, alerting both patient and the dentist about the checkups whenever any genetic or lifestyle information indicates increased susceptibility to dental diseases, managing and maintaining record $\mathrm{AI}$ integrated with $\mathrm{CBCT}$ and MRI helps in detecting minute deviations; in orthodontics the model helped in pointing out the contraindications while presenting in the data to assess the craniofacial skeletal and dental abnormalities.

Clinical decision support systems (CDSS) are computer programs that help analyse patient data and make decisions regarding diagnosis, prevention and treatment. They can generate alerts on potentially dangerous condition of the patient. But the substantial cost of knowledge acquisition and knowledge maintenance remains the most challenging problem for the sustainability of CDSSs. ${ }^{7}$ Finally, it can be concluded that, AI has benefitted the healthcare industry in certain ways. However, further research and advancements in the field are required. To what degree we are dependent on AI is a question as of today. As long it is made use in "lifeless" issues it is not risking. However, when it comes to its application in human or any other forms with life and vitality, there needs further studies to be researched, repeated and recommended for its application in medicine or dentistry.

\section{References}

1. Russell SJ, Norvig P, Davis E. Artificial intelligence: a modern approach. 3rd ed. Upper Saddle River, NJ: Prentice Hall. (2010).

2. "Golden years" of AI (successful symbolic reasoning programs 1956-1973).

3. Weighing in on the pros and cons of artificial intelligence. https://techspirited.com/pros-cons-of-artificial-intelligence; accessed on Mar 17, 2018.

4. Alfred NG (7 August 2016). "IBMs Watson gives proper diagnosis after doctors were stumpted". NY Daily News. Archived from the original on 22 September 2017. 
5. Khanna SS, Dhaimade PA. Artificial Intelligence: Transforming Dentistry Today Indian J Basic and Applied Med Research; June 2017: Vol. - 6 (3), 161-167.

6. Khanna S. Artificial intelligence: contemporary applications and future compass. Int Dent J. 2010 Aug; 60(4):269-72.

7. Mendonca EA. Clinical decision support systems: perspectives in dentistry. J Dent Edu. 2004 Jun; 68(6):589-97.

KL Vandana

Senior Professor

Department of Periodontics, College of Dental Sciences

Davangere, Karnataka, India

NB Nagaveni

Professor

Department of Pedodontics and Preventive Dentistry, College of Dental Sciences

Davangere, Karnataka, India 